Artículo de reflexión

Cómo citar: Colonia, O. (2019).

Innovación didáctica. Acción precipitada o reflexionada. Praxis Pedagógica,

19(24), 9-31. http://dx.doi.org/10.26620/

uniminuto.praxis.19.24.2019.9-31

ISSN: 0124-1494

eISSN: $2590-8200$

Editorial: Corporación Universitaria Minuto de Dios - UNIMINUTO

Recibido: 26 de febrero de 2019

Aceptado: 04 de abril de 2019

Publicado: 5 de junio de 2019

Conflicto de intereses: los autores han declarado que no existen intereses en competencia.

\section{Innovación didáctica}

\section{Acción precipitada o reflexionada}

\author{
Educational innovation \\ Precipitated or thoughtful action
}

\author{
Inovação didática \\ Ação precipitada ou atenciosa
}

Oscar Colonia Alcalde

\section{Resumen}

En las instituciones educativas hoy en día se plantea un componente que prácticamente ha sido ajeno y un poco extraño en el aula de clases en el desarrollo y potenciación del oficio y profesionalismo del maestro, tal como lo señala Colonia (2008 a) en un post de su blog Innovaciones y Ensayos en la Escuela, en la publicación Hernández (2012) en un post de su blog Diálogo entre profesores y en la publicación de Hernández (2015) en un post del blog Lo que debes saber de la educación especial; cómo es la práctica de investigación formativa, que permita evidenciar los logros y cuasilogrados de los actores en el aprendizaje de los saberes y conocimientos escolarizados que circulan en el aula de clases.

Bajo esta perspectivas es urgente que emerjan en el accionar diario del maestro instrumentos pedagógicos como: la autobiografía, el diario pedagógico que le posibilitan la emergencia de herramientas didácticas que estructuren la transformación e innovación en el aula, que desarrollen en los encuentros nuevas formas de abordaje del saber, para lo cual, se plantea en la innovación didáctica desde la matemática los siguientes pilares: un dibujo, referente para el desarrollo conceptual y procedimental, desde lo histórico y desde lo social; la relatoría, considerada como la caja negra de la innovación, donde se registran los aconteceres y posibles acontecimientos que surgen de la cotidianidad de cada encuentro, ella se segmenta en secciones para diferenciar las actividades y las estrategias pero se integran para darle sentido al aprendizaje desde diferentes marcos conceptuales (secciones); la autoevaluación 
que permite transformar una evaluación desde la nota, a una evaluación por el saber apropiado y la maqueta, posibilita que el aplastamiento del mundo, se vea en dimensión real y proporcional. Con esta estructuración metodológica, se pretende una activa acción en cada encuentro y desde los registros, una práctica de investigación con profundización.

Palabras clave: innovación educacional, comunicación interactiva, investigación pedagógica.

\begin{abstract}
In the educational institutions today there is a component that has practically been foreign and a little strange in the classroom in the development and empowerment of the profession and professionalism of the teacher, as is the practice of formative research, which allows To demonstrate the achievements and cuasilogrados of the actors in the learning of the knowledge and schooled know-how circulating in the classroom.

Under this perspective it is urgent to emerge in the daily actions of the master pedagogical instruments such as: The Autobiography, The Pedagogical Diary, which enables the emergence of didactic tools that structure the transformation and innovation in the Classroom, to develop in the meetings, new ways of approaching knowledge, for which, is posed in didactic innovation from mathematics, the following pillars: A drawing, a reference for conceptual and procedural development, from the historical and from the social: The Rapporteurship, Considered as the black box of innovation, where they record the events and possible happenings that arise from the daily life of each meeting, she is segmented into sections to differentiate activities and strategies but are integrated to give Meaning to learning from different conceptual frameworks (sections); The self-assessment, That allows to transform an evaluation from the note, to an evaluation by the appropriate knowledge and The model, allows the crushing of the world to be seen in real and proportional dimension. With this methodological structuring, it is intended an active action in each meeting and from the registers, a practice of investigation with deepening.
\end{abstract}

Palabras clave: educational innovation, interactive communication, pedagogical research.

\title{
Resumo
}

Nas instituições educativas hoje em dia se propõe um componente que praticamente tem sido alheio e um pouco estranho em aula de desenvolvimento e potenciação do ofício e profissionalismo do professor, tal como apresenta Colonia (2008 a) em uma postagem de seu blog Inovações e ensaios na escola, na publicação de Hernández (2012) em uma postagem de seu blog Dialogo entre professores e na publicação de Hernández (2015) em uma postagem do blog O que deves saber da educação especial; como é a pratica da investigação formativa, que permite evidenciar as realizações e quase realizações dos atores na aprendizagem dos saberes e conhecimentos escolarizados que circulam na salas de aula. 
Sob esta perspectiva é urgente que surjam nas ações diárias do professor instrumentos pedagógicos como: a autobiografia, o diário pedagógico, que possibilitam a emergência de ferramentas didáticas que estruturem a transformação e inovação na aula, que desenvolvem nos encontros, novas formas de abordagem do saber, para o qual, se propõe na inovação didática desde a matemática, os seguintes pilares: um desenho, referência para o desenvolvimento conceitual e procedimental, desde o histórico e desde o social: o relator, considerado como a caixa negra da inovação, onde se registram os acontecimentos e possíveis acontecimentos que surgem do cotidiano de cada encontro, se segmenta em seções para diferenciar as atividades e as estratégias, mas se integram para dar sentido a aprendizagem desde diferentes marcos conceptuais (seções); a autoavaliação, que permite transformar uma evacuação desde a nota, a uma evacuação pelo saber apropriado e a maquete, possibilita que o esmagamento do mundo, se veja em dimensão real e proporcional. Com esta estruturação metodológica, se presente uma ativa ação em cada encontro e desde os registros, uma prática de investigação com aprofundamento.

Palavras chave: inovação educacional, comunicação interativa, investigação pedagógica.

\section{Importancia de la innovación para optimizar la reflexión}

Es necesario que la propuesta de innovación deje claro que sus fundamentos y el diseño de las herramientas como el dibujo, la relatoría, el diario y la maqueta utilizadas en la propuesta, no son determinantes en lo metodológico y didáctico. Igualmente, el maestro decide sobre la implementación y su desarrollo, de acuerdo con un contrato didáctico explícito (Colonia, 2008 b).

Es importante que la innovación se genere desde la práctica, pero es necesario relacionarla con la teoría, posibilitando con ello, la fluidez en la interpretación y no se torne intraducible. De la misma manera, innovar dimensiona los estados de tensión que se dan en el ámbito escolar, lo cual implica cuestionar las normas para actuar y dar las pautas para pensar. Llevando a los actores de la escuela a experimentar una fuerte resistencia a los cambios y transformaciones del saber y su comunicación (Colonia, 2008 b).

Por último, pensar que la innovación en sí misma transformará el contexto escolar es entender a los maestros como incompetentes, reduciendola a un activismo. A diferencia de lo anterior, quien innova, en el sentido real del término, corre el riesgo de 
ser satanizado toda vez que sus acciones irrumpen las prácticas tradicionales y buscan que los alumnos piensen y creen, generando una simbiosos entre la destreza y la habilidad (Colonia, 2008b).

\section{Reflexiones acerca de la conformación estructural de la propuesta de innovación a partir de la teorización}

El ejemplo del núcleo familiar con un adecuado ambiente académico, los encuentros vivenciales con maestros de universidades del Valle, Antioquia, Nacional, entre otras, generaron inquietudes para acercarme más a la Didáctica y la Pedagogía. Pensaba que la matemática se enseñaba de manera individual, pero, a partir de mi experiencia en el aula, reconocí que es posible enseñar matemática a través de una ingeniería didáctica (cooperativa) (Colonia, 2008c).

Es importante señalar que la actividad matemática es impensable sin la conceptualización de variable, que es una de las tantas reflexiones generadas en los encuentros con maestros versados en la Didáctica, en los que se resalta que ésta se debe apropiar desde el lenguaje. Esta comprensión de la didáctica desde el lenguaje Ilamó mi atención porque es componente fuerte de la propuesta, lo cual permite por un lado, la comunicación de los saberes y por otro lado, la representación de los mismos. Estos encuentros me permitieron analizar que, la literatura, desde la educación matemática, es un elemento movilizador de las mismas. Desde esta mirada, el uso en la matemática de la metáfora literaria puede desplazar su significado, en cambio, hablar de la metáfora matemática garantiza fundamentalmente su contextualización y comprensión (D’Amore y Fandiño, 2002).

A mi modo de ver, el uso de metáforas es una aproximación a la transposición didáctica que Colonia comprende

al ser designado como saber que se enseña un contenido de saber, sufre, desde ese momento, un conjunto de transformaciones adaptativas que lo vuelven apto para tomar un lugar entre los objetos de enseñanza, es decir, el trabajo pedagógico que transforma un objeto de saber en un objeto de enseñanza, se la Ilama transposición didáctica (2007a; 2007b). 
Entre tanto, es necesario que el maestro al hacer una antropología didáctica, pueda acopiar los saberes que circulan en la escuela con el propósito de que la transposición didáctica permita una más y mejor apropiación del saber matemático, con lo cual se puede afirmar en relación con los estudiantes:

- Son distintos y saben hacer cosas distintas.

- Porque lo que se propone en clase debe partir de lo que supuestamente saben.

- Al ser distintos, usarán lenguajes distintos o pueden utilizar los lenguajes de modo distinto, entonces se requiere que la escuela propicie herramientas que le posibiliten comunicar y elaborar sus propias experiencias, que desarrollen su propio saber y además debe estar pronto a que estos instrumentos permitan interpretar y traducir los avances de cada uno (Relatoría).

Es claro que la propuesta de innovación busca desde su estructura externa e interna la diferenciación del aprendizaje en cada una de las secciones de la Relatoría, de esta manera, se pretende: atenuar y flexibilizar las tensiones, las asimetrías y las dificultades que se generan en la diversidad del aula, partiendo de una singularidad sustentada en la dinámica intuitiva-deductiva, permitiendo, desde la conjetura, la configuración de posibles constructos o conceptos.

En este sentido, la presente propuesta que se distingue de la escuela academicista, sin embargo, Ilama la atención sobre lo que se escribe en el siglo XIX Gustavo Le Bon, Citado por Giordano, \& Vecchi

hay pues ciertas cosas que deben ser modificadas en la instrucción tal y como se desarrollan hoy en día. Las instituciones y las costumbres han cambiado, las ciencias han progresado a pasos agigantados, sólo el fondo de la enseñanza no ha cambiado, tal y como era hace un siglo, así nos encontramos hay en día. Hay que terminar de una vez por todas con esta instrucción de catálogo que lo toca todo y no profundiza en nada, con esta educación enciclopédica que sobrecarga la memoria sin desarrollar el pensamiento y que no deja tras ella sino una fatiga a menudo irreparable y una aversión casa insuperable por el trabajo intelectual (1988, p. 2). 
Divisar en retrospectiva su estructuración, permite, mirar y analizar los pilares fundamentales de la Innovación Matemática que soportan la transformación de la acción, para lo cual, es necesaria una mirada antropológica, que posicione y consolide su participación en la perspectiva didáctica y proyecte, de manera pragmática, cada una de las herramientas propuestas, posibilitando la acción y la investigación.

Por lo anterior, es importante presentar el método realizado en una actividad de innovación matemática. Primero, se hizo un análisis complementario al Dibujo, que consiste en un paisaje, realizado en un plano cartesiano. Es de aclarar que el diseño de esta herramienta propicia el trabajo colaborativo con el profesor de artística, quien no debe trabajar suponiendo que los estudiantes adquirirán el máximo grado de desarrollo de la perspectiva. En este sentido, la innovación matemática brinda diferentes formas (perspectivas) de contemplar el mundo.

Partiendo de la apuesta colaborativa mencionada anteriormente, se comprende al estudiante como un sujeto activo del proceso de interaprendizaje, es decir, reconocer al estudiante como poseedor de un saber, por tanto, "la interacción maestro-estudiante, se deben entender como el diálogo de dos discursos, el encuentro de dos saberes o de dos verdades" (Carlos Federici).

En el desarrollo y en el contexto de la propuesta de innovación, se pueden apreciar rasgos del dibujo del estudiante, que coinciden con la geometría descriptiva. También se puede observar tanto la simultaneidad temporal (es decir varios acontecimientos sucedidos en tiempos distintos, representado en un mismo tiempo), como la desproporción subjetiva (el tamaño de los objetos del paisaje depende no de sus medidas reales, sino de su significación personal).

Estas apreciaciones se apoyan en tres aspectos: la historia del dibujo, los modelos piagetianos articulados a la reflexión psicogenética de la historia de la geometría y un tercer aspecto "Las lecturas de lo obvio" denominadas así, por las investigaciones de la pintora Beatriz González.

Agregando a lo anterior, se puede decir que en la "historia del dibujo" algunas investigaciones muestran la relación de los dibujos de los estudiantes con las formas ya elaboradas en otras 
épocas y por otras culturas, es claro que la ontogénesis no siempre repite la historia del ser humano, se podría establecer algunas contradicciones, cuando por ejemplo, la topología es la primera en aparecer en el niño, pero es la última en conceptualizarse en la escuela.

Cabe señalar, según investigaciones de Mariño que los códigos para representar el espacio por parte de los estudiantes son similares a los usados por los pintores del siglo XX, los cuales, a su vez utilizan parámetros análogos a los pintores anteriores al siglo XV (fecha en la cual se creó la perspectiva), de ahí se desprende que la perspectiva es una arbitrariedad que busca representar la tercera dimensión, en relación con el espectador, creándole la idea que se encuentra ahí, es decir, como "vista clara" lo que pretende es hacer creer un tiempo y espacio como reales en la imaginación real del que la aprecia. Lo anterior, permite sospechar que la perspectiva es una "objetividad mentirosa", ya que el espectador mira la realidad sólo desde el punto de vista del dibujante, que sería análoga a la comunicación, casi en una sola vía entre el maestro y el estudiante. En general, con esta reseña de estudios sobre el dibujo, que en la propuesta de innovación se conforma con un paisaje (Referencia Contextual), intersectada con un sistema de Referencia (plano cartesiano y cuadrícula), que posibilita una conjugación entre lo matemático y lo estético, lo matemático y lo histórico, lo matemático y lo antropomórfico y otras conjunciones que hacen ver este pilar (El Dibujo) como una herramienta didáctica en el aula pero a su vez un objeto de estudio permanente.

Se continua con el análisis complementario de la Relatoría, que es medular en la Innovación matemática, por ser una herramienta que posibilita el registro de los "relatos" que se dan en la lectura de las teorías, conceptos matemáticos y personajes que aportan al crecimiento del saber matemático, la lectura que se debe hacer de los aportes del maestro en el desarroIlo de la unidad temática, la lectura que se debe hacer de los aportes de cada uno de los pares en el desarrollo de la unidad temática, la lectura que se debe hacer de los gráficos en el sistema de referencia (plano cartesiano) y las diferentes situaciones que se derivan, para lo cual se debe tener en cuenta la organización estructural del relato y a su vez se pueda dar una ilación de tipo argumental que muestre las dificultades y los avances en el proceso comunicativo. 
Cabe señalar, que en el desarrollo de la Experiencia, se aprecia que una de las grandes dificultades de un relato, es el proceso de ilación de las palabras (sintáctico) que a veces muestra una serie de trazos o párrafos que no permiten coherencia y consolidación argumental. Es interesante estudiar que tanto la lectura, como la escritura de un relato, generalmente los estudiantes y los adultos no siguen una estructura estándar de éste, sino por el contrario tratan de leerlo o construirlo (escribirlo) de acuerdo a unas ideas previas, situaciones o intereses. Esto es, según la perspectiva constructivista, la reaparición en años recientes tanto en el campo de la lingüística y de la teoría cognitiva, el relato, como el estudio de las concepciones de los niños y jóvenes.

De manera análoga, el aspecto teórico está relacionado con la forma de las reglas usadas para describir el relato. Por ejemplo, la hipótesis anterior, lo que plantea es que estas reglas generan una estructuración jerárquica, que se evidencia en la sintaxis que relaciona la estructura básica de Chomsky (1965), se podría afirmar que si tomamos la Unidad Temática como un relato, que formula, plantea, escribe y desarrolla el estudiante y que este relato (Unidad Temática), está dividido por secciones, que hacen que se pueda determinar por parte del estudiante, lo que para él es más importante y cuáles no son tan importantes, por ejemplo (Fundamentación Teórica- procedimientos matemáticos- proposiciones- creatividad- autoevaluación, conclusiones). Lo anterior podría establecer que el estudiante considere cuatro secciones importantes (episodios) y las otras menos importantes, lo cual lleva en muchos casos a la reducción del relato, de acuerdo a esta jerarquía subjetiva del estudiante, lo anterior permite describir unos acontecimientos importantes al lado de otros menos importantes.

Se observa en los procesos investigativos alrededor del relato, que para el estudiante, ocupa más tiempo y obviamente más desarrollo en el mismo lo que es significativo en el día a día, y deja más superficial y adjetival lo que el considera complejo y poco significativo para el desarrollo de su proyecto, permitiendo a veces hacer redireccionamiento al relato, para evitar que lo fundamental o sustantivo pase a un segundo plano y se pierda el propósito esencial de la comunicación matemática por medio de un argumento coherente en la rigurosidad de esta disciplina. La mayor parte de estudiantes acaban descubriendo que pueden leer y escribir para entretenerse, informarse y evadirse de la realidad o simplemente para preparar una evaluación. 
En el desarrollo del relato, el estudiante en la acción didáctica se apropia de un componente epistemológico que permite hacer un recorrido teórico, histórico y filosófico de los matemáticos y no matemáticos que le aportan a los conceptos que se abordan en la unidad, es importante tener en cuenta, que éste se intersecta en el relato con un componente cognitivo que debe establecer lo semiótico, es decir, la concepción general y lo semántico donde se plantea lo particular del relato, es decir, en la relación de lo general (semiótico) a lo particular (semántico), se debe establecer procesos hipotético-deductivos que posibiliten extrapolar las teorías planteadas en la Sección de Fundamentación Teórica, en la sección de Procedimientos Matemáticos, donde el estudiante a partir del dibujo visualiza los procedimientos, los describe, luego los analiza e interpreta a la luz de las teorías y conceptos que fundamentan la unidad, permitiendo con el relato, relacionar los objetivos matemáticos observados, con lo representativo y observable en el dibujo, generándose una ampliación de la descripción, utilizando el razonamiento que permita desde la teoría hacer el análisis, interpretar con las conjeturas e hipótesis y generar un argumento con validez matemática.

El desarrollo de estas acciones deductivas, determinan el desarrollo de estrategias que generan la formación del pensamiento matemático. En el desarrollo del relato en la Unidad Temática y luego en las unidades temáticas que configuran el módulo de matemáticas o Relatoría (producción textual), el estudiante se involucra en una actividad intelectual que tiene una consecuencia posterior que es la disponibilidad de ese saber en un doble status: como herramienta, cuando la utiliza en la interacción en el Dibujo y su propio relato y como objeto de estudio cuando por medio de sugerencias y aportes que se hacen en la evaluación formativa, le permite desarrollar un estudio y una práctica investigativa sobre los avances y dificultades en el desarrollo de la Unidad Temática.

En general, la relatoría en cada unidad de innovación, determina la construcción de sentido desde los procesos y saberes matemáticos, permite la producción del mundo simbólico desde la estructuración semántica y sintáctica del lenguaje, la reconstrucción del camino recorrido en la Unidad Temática y en la unidades temáticas permitiendo prescribir en muchos casos la simplificación conceptual con la unificación de lo diverso (reducción), la separación de conceptos que desde su estructura permanecen ligados (disyunción) 
En el Relato se pueden apreciar las nociones que se generan en procesos de lectura y escritura de Teoría Matemática, planteamiento de hipótesis desde la visualización y experimentación (Dibujo- maqueta), procesos generadores de nociones, la construcción de proposiciones desde la sintáctica y la rigurosidad matemática, el proceso de análisis y síntesis que ayudan al estudiante a la elaboración de conclusiones generales de la unidad, estableciendo en últimas que las nociones son el corazón del saber matemático.

Continuando con la estructura de la propuesta de Innovación Matemática se aborda la Maqueta, que se puede considerar la proyección de la observación. Hoy en día las matemáticas se enseñan como una asignatura de apoyo a la elaboración de estructuras e instalaciones, por lo tanto, se requiere generar a partir de la geometría analítica clásica, bidimensional y tridimensional, enfoques didácticos y pedagógicos que permitan establecer líneas de fuga, líneas en verdadera longitud, ángulos de proyección, proporciones que permitan la construcción de un modelo desde los dibujos que muestran el aplastamiento del mundo según el estudiante, como lo afirma Colonia (2008 a) en su Blog Innovaciones y Ensayos en la Escuela. Es cierto que los productos, por ejemplo en la arquitectura son elaborados por el hombre, así como también las matemáticas son obra del hombre. Pero se puede apreciar que están en dos planos distintos: la matemática generalmente trabaja en espacios y conceptos abstractos; mientras que la arquitectura y el diseño que es la vez técnica y arte, se ocupan del espacio concreto, del espacio con relación al hombre que lo habita, a sus necesidades, a sus intereses, a sus costumbres y a su posible cultura. Entonces, para que la matemática contribuya al desarrollo y proyección de lo bidimensional a lo tridimensional, no sólo se requiere desestructurar los programas de matemáticas, sino fundamentalmente cambiar el enfoque con que se la enseña para que el aprendizaje sea más significativo y con sentido pragmático, la maqueta como reflejo y proyección de un plano (Dibujo con cuadrícula), busca acercar los trazos y proyecciones geométricas en la realidad tridimensional, para lo cual se requiere la interpretación de la proyección, perspectiva, la jerarquización de los objetos en el dibujo, la dimensión y referencia espacial, la proporción de la longitud, área y volumen que se proyectan en los objetos y su relación con los objetos matemáticos como lo afirma Colonia (2008 a) en su Blog Innovaciones y Ensayos en la Escuela. 
La Maqueta es un modelo que se transforma en la medida que se modifican y cambian los objetos o espacios en el dibujo con cuadrícula, permitiendo vivenciar y experimentar los cambios y transformaciones de acuerdo a las necesidades, intereses y problemas en el desarrollo de la interacción matemática.

Se procede hacer una reflexión alrededor del Diario Pedagógico o una Autoevaluación con formación, que es una herramienta pedagógica que permite conocer lo vivencial y experimental de cada estudiante, generalmente se parte del desarrollo conceptual, procedimental y actitudinal, permitiendo conocer los niveles de apropiación en estos componentes por parte del estudiante que deben reflejar el sentido de pertenencia, de identidad y de pertinencia con relación a su proyecto.

En esta herramienta se debe plasmar los aportes, expectativas y reflexiones, de cada estudiante, partiendo de un título creativo y significativo que refleje la apropiación filosófica y conceptual de su proyecto, que se hace en relación con el saber, con el hacer y con el saber-hacer. Con los aportes de estas herramientas didácticas que configuran una propuesta metodológica para el aprendizaje y enseñanza de las matemáticas se podrá a partir del trabajo práctico, permitir el cambio conceptual, buscar con la didáctica como saber el mejoramiento de la comprensión a nivel conceptual y, además, acercar la teoría a la práctica por medio de la resolución de problemas, generando prácticas de investigación.

Acuerdos y proyección desde la acción. Cuando se da una transformación de los procesos en el aula de clases, es necesario presentar en forma explícita, las herramientas didácticas que posibilitan el cambio, con un propósito esencial, como es la apropiación de ella, que permita el desarrollo progresivo de las actividades y estrategias que conforman la estructura interna y externa de la Innovación.

Las cuatro herramientas didácticas o pilares que soportan la propuesta, tienen propósitos específicos que determinan el aprendizaje con una gran aproximación a la significación.

Es importante recalcar que el maestro en formación y el maestro formador, con estas herramientas didácticas pueden tener aproximaciones importantes al pensamiento abstractivo que les permite aligerar, avivar y despertar el espíritu y la conciencia científica en los niños, de tal manera que les permitan un tránsito amistoso al espíritu y la conciencia científica y matemática, 


\section{experimentando la urgencia de poder hacer la reconstrucción} científica en el aula, partiendo del pensamiento concreto de los niños generando la comunicación en el aula de una unidad del mundo, (mirado holística) y a su vez que los niños comprendan la diversidad de las cosas, permitiendo que fluya la pregunta a partir de construcciones acordadas en el aula, puede notar que el espíritu o motivación a saber considera el conocimiento como todos aquellos que se pueden considerar más cercanos a las necesidades, a los intereses, y a los problemas comprometidos en el contrato didáctico, lo cual puede generar en el maestro formador y en formación, la posibilidad de comunicar opiniones que traducen esas necesidades, intereses o problemas, en conocimientos que al plantear como idea dominante, eta podrá polarizar el espíritu o motivación en situaciones que no se podrían considerar problemáticas en un ámbito escolar, lo cual conduce a pensar que nuestro espíritu o motivación como maestro, o comunicador en el aula, preferirían lo que conforma y se podría decir lo que corrobora un saber al que lo contradice o lo falsea, lo cual puede explicar de alguna manera por parte de los actores en el aula, tal vez una doble vía, la preferencia a las respuestas más que a las preguntas o aún más, la predilección de respuestas sin preguntas.

Se puede señalar a partir de lo anterior que la intersección de las herramientas didácticas que estructuran la innovación matemática, posibilitan desde una situación didáctica, la formulación y desarrollo de actividades en cada una de las secciones que conforman la unidad formativa de la tal manera que en la Sección \# $\mathbf{1}$ se elabora un mapa conceptual, con los referentes y conceptos que se abordaron en el período académico, de tal forma que al construir el mentefacto, se le traza una ruta conceptual, que permitirá el desarrollo del ensayo, que posibilite por medo de un título sentí-pensante, el englobe de los conceptos que se abordan en la unidad, relacionados con los referentes teóricos, permitiendo en la producción textual, la aproximación de la lógica de las cosas, con las cosas de la lógica. Cabe pensar que con lo anterior, en el Sección \# 2, de procedimientos matemáticos, se optimiza por medio de una situación problemática, en la cual se plantean problemas o situaciones que le permiten al alumno, movilizarse por diferentes marcos conceptuales, registrar lo general de los procesos y luego discriminar y particularizar, de tal manera que realiza un proceso hipotético-deductivo y viceversa. En estas situaciones para enseñar que se planean en las estrategias de la situación 
problemática, en la cual al estudiante se le asignan varias tareas, con el propósito de resolver problemas, pero para poder acceder a éstas situaciones, se requiere la comprensión de preguntas y de la información que se comunica en la clase por el maestro, aquí se puede establecer que los hábitos del maestro esperados por los estudiantes y a su vez, los comportamientos de los estudiantes, esperados por el maestro, conforman el contrato didáctico (Brousseau 1980).

Generalmente en el abordaje de la solución de un problema, los estudiantes tienen dos aspectos muy claros como son: leer y releer el problema y estar tranquilos y razonar por sí solos denotando lo anterior, normas explícitas de un contrato comportamental, como es trabajar por sí solos y mediante sugerencias del maestro, como es leer y releer el texto del problema.

Es bueno advertir que muchos de los acuerdos explícitos como: la situación problemáticas, la secuencia didáctica y cronograma de entregables que regulan la acción en el aula de la innovación, hay otros que no son explícitos y a veces inexistentes, pero creados por los imaginarios de los actores escolares, con el propósito de darle cohesión a su vida en la escuela, pero que se tratan de obviar en este proyecto, con el fin de poder visibilizar en una forma más clara, los avances y dificultades en el desarrollo académico y didáctico del saber enseñado y aprendido. Es claro que en este proceso de la práctica se registra una particular argumentación que evidencia las formas de pensar y actuar de los estudiantes, de sus propios acuerdos en el equipo cooperativo y la manera cómo se interactúa entre los actores como: los estudiantes, el maestro y el propio saber que circula y edifica en clase.

Ahora, cuando el estudiante en un momento determinado dice "no entiendo", no quiere decir que fracasó rotundamente el diseño estructural de la innovación, posibilita y abre oportunidades de flexibilizar procesos muy ortodoxos y desde las secciones, abre perspectivas de apropiación del saber.

Se podría colegir, mirando lo anterior que en el desarrollo de una clase de matemáticas, hoy en día, se busca que los alumnos se apropien de las estrategias para plantear y solucionar un problema, lo cual implica que un momento dado, este proceso se mecaniza y los estudiantes no "aprenden matemáticas" sino que utilizan métodos que al memorizarse son como obstáculos propios en la mirada de resolución de otros problemas que no corresponden al "ejemplar". 
En la estructura interna de la innovación se busca por diferentes vías, enseñar las matemáticas, posibilitando el actuar, el formular y el probar, que se puede modelar y conformar con la teoría de las situaciones didácticas (Brousseau 1996).

Las situaciones didácticas en el contexto de la innovación matemática, busca acercarse a un alto porcentaje de la situación de enseñanza en el aula, tratando de atenuar dos asimetrías que se evidencian como son: una asimetría epistémica, en la cual, el maestro a partir de las estrategias formuladas en cada sección que conforma la unidad formativa, proyecta al alumno hacia una apropiación de saber matemático permitiéndole circular por diferentes marcos conceptuales con el propósito del el establecimiento de relaciones con otros saberes y otros referentes que permitan la secuenciación y la significación en otros contextos. Por otro lado, la otra asimetría, es inversa al anterior, es decir el maestro espera tener la confianza del estudiante con el propósito de potenciar las estrategias formuladas y acercarlo al aprendizaje efectivo, pero en este caso, no ha sido posible un respaldo institucional que lo posibilite, pero la resistencia de actores institucionales, aunque no posibilitan incrementar la confianza del alumno, ha permitido que la resistencia mecánica, sirva como una utopía, que cada día lleva a la innovación a seguir caminando con más razones y condiciones académicas que han determinado que por razones obvias, sus herramientas didácticas, sus principios, su estructura externa e interna sea valorada y validada por la comunidad académica nacional e internacional, permitiendo un avance significativo en sus relaciones y proyecciones conceptuales.

En esta estructura metodológica de la innovación se busca romper con el contrato didáctico bien sea explícito o implícito, es decir que además de proponer estrategias y formas para la resolución de problemas, se busca que al relacionar la teoría y los conceptos a su hacer pueda comprender no sólo un problema específico, si no que promueva en si equipo cooperativo, las diferentes formas o perspectivas en su solución.

Desde éstas múltiples perspectivas que promueve la experiencia, es necesario darle la mirada a la Sección de Creatividad, que busca desde el juego y desde la lúdica acercar a muchos estudiantes al saber matemático escolar, entonces, se tiene que aceptar que en muchos casos los errores en la escuela son de percepción que es la clave en el desarrollo y potenciación del 
pensamiento. En los procesos creativos se modula la experiencia, en procesos creativos, los alumnos ven y se apropian de los conceptos con una lente de gran ángulo que hace que los conceptos se trasladen a otros contextos, se puedan mirar otras explicaciones, cuando el estudiante construya los acrósticos, sopas de letras, crucigramas, grafitis, juegos, etc., posibilitando que en la construcción de estas actividades, pueda evocar, investigar, relacionar, tomar perspectivas con poca aproximación matemática, pero que al consolidarla muestra un producto que evidencia: cambiar, transformarse y muchas relaciones a nivel conceptual y procedimental. En la sección de creatividad intersectada con otras cinco secciones se pretende que los actores en el aula, especialmente el alumno, potencie el pensamiento con el propósito de obviar y marginar actitudes propias de la escuela tradicional como: actuar puramente por instinto, repetir las rutinas de costumbre, hacer lo que otra persona decide y ordena y dejarnos llevar por la emoción del momento.

Entonces, la creatividad, además de ser alternativa cuando se aborda una acción o un concepto, se debe tener también el componente artístico, es así, que este matiz artístico, posibilita la organización estructural del concepto o procedimiento que se desarrolla. Es por ello que De Bono, acuña el concepto de pensamiento lateral, para determinar cambios o transformaciones en conceptos y percepciones, permitiendo que a partir de un concepto central, se pueda generar otros alternativos o subconceptos que se derivan en forma literalmente lateral, es como en el desarrollo de las actividades planeadas y formuladas en la Secuencia Didáctica, se puedan ensayar diferentes percepciones, conceptos y enfoques en lugar de trabajar más fuerte con los tradicionales.

Con las múltiples actividades en esta sección, se pretende que los actores en clase desarrollen la inventiva, la imaginación, el pensamiento divergente, de tal forma que se puedan plantear: conjeturas, nuevas hipótesis que resuelvan problemas, utilizando múltiples caminos o lugares epistémicos propios, con soluciones originales, desde luego, que se puedan comprobar en diferentes marcos conceptuales, conduciendo a una diversidad metodológica que sobrepasa la unicidad, para aproximarse a la unidad de procesos.

Existen muchas técnicas para desarrollar e incrementar la capacidad creativa como: la lluvia de ideas en clase o en grupos, 
los mapas mentales (Sección \# 1), la selección de ideas (Sección \# 2 y 4), la clasificación de ideas (Secciones \# 1, 3, 4) los mapas conceptuales (Sección \#1), etc. Es claro que la creatividad es la forma de diversificar los caminos en la solución o creación de un problema, que se podría acercar a la idea de creatividad del psicoanalista Ernst Kris, que la asocia, como la habilidad en encontrar nuevas conexiones entre ideas, es decir conectando el pensamiento consiente con el inconsciente. En esta sección de la unidad formativa se la innovación, invita a los actores escolares, en especial al alumno, a estar en una continua búsqueda que lo mueva a crear puentes con su imaginación.

Es necesario dentro de la democratización de una secuencia didáctica, poder acercarse y pasar de la creatividad a un pensamiento lógico proposicional, donde se pueda conformar desde los conceptos y desde los referentes, frases que determinan falsedades o verificaciones de una estructura conceptual o procedimental a nivel matemático, de tal forma que los elementos más simples representan proposiciones y cuyas constantes llamadas conectores lógicos, representan operaciones sobre proposiciones, que a su vez, pueden configurar otras proposiciones con un alto nivel de complejidad.

Es claro que en la lógica proposicional se requiere determinar si una proposición es falsa o verdadera y para lo cual requiere una construcción sintáctica que determine una fluidez en su configuración. Una palabra aislada o un elemento matemático que no esté en contexto o situado matemáticamente no se podrá evaluar y mucho menos plantear una argumentación con un buen sustento matemático y científico. En esta sección se pueden formular proposiciones atómica, es decir, que es una sola frase que no tiene un conector u operador, por otro lado cuando son moleculares, se trata de dos proposiciones que están conectadas por un operador. Cabe señalar que las proposiciones que se construyan tienen un alto porcentaje de conceptos abordados en el período académico, que requieran los estudiantes conectarlos o relacionarlos de alguna forma, con el propósito de argumentarlos y contra-argumentarlos desde la evaluación de falsedad o veracidad.

Es importante en esta sección, inducir a los alumnos en el uso de los tres lenguajes en matemáticas como son: el gráfico, el cotidiano y el simbólico, tanto en la formulación como en la argumentación y contra-argumentación de dichas proposiciones. 
Si se mira este fragmento de la unidad formativa se podría afirmar que en el enmarque fenomenológico y en especial la crítica a la vía lógica, todas las tareas de la lógica formal y trascendental pueden resumirse bajo un único proyecto y es e de llevar a la luz de la razón que subyace y es latente a todo saber y a toda ciencia, incluida la fenomenología. Aquí, en este proceso innovador, no se describe únicamente la experiencia muda y sin reflexión, que antecede a toda operación de categorización y de raciocinio, sin que trata de profundizar en esa grieta o rendija que siempre queda entre la experiencia y la razón, entre el mutismo y la palabra, se trata entonces, de comprender el origen de la razón que se introduce en la experiencia o la experiencia que se comprende en la razón. Se puede decir que toda ciencia es, en primer lugar, un conjunto de proposiciones o jui$\operatorname{cios}^{1}$ o también se podría decir, toda ciencia, es un conjunto de significaciones y de combinaciones de significaciones, ya que estas se dan al interior de las proposiciones, además, si éstas constituyen una ciencia matemática, no pueden ser contradictorias, es decir sus elementos -proposiciones atómicas-moleculares y los operadores- deben ser compatibles entre si. Se busca en esta sección desde la crítica de la vía de la lógica en el enmarque fenomenológico, a partir de combinaciones de disciplinas complementarias de la propia matemática, conformar desde un sistema consecuente de proposiciones fundadas y configuradas, unas en otras, posibilitando la potenciación de posición propositiva y argumentativa de los actores en el aula, primordialmente el alumno.

Al llegar aquí, se puede mirar la Sección \#5 de la innovación que pretende transformar la autoevaluación del aprendizaje, en el sentido de no mirar la parte profesionalizante o de notas sino, establecer lo que se aprende en la Unidad formativa, con relación al saber, al hacer y la intersección de ellos, para la aplicación y derivación del aprendizaje en otros contextos.

Examinando la autoevaluación como una interpretación de la vía psicológica en el marco fenomenológico, se orienta a la exploración de las configuraciones de las vivencias, intenciones y sus atributos y sus desarrollos en la búsqueda de una descripción integral, enfatizando en la dimensión intersubjetiva colocando "la experiencia de lo ajeno" en la base de todo planteamiento y no como un estudio que sea preciso agregar para "salir de los

1 Lógica formal y trascendental. Edmund Husserl pag. 152. 
límites"; de este la vida comunitaria y en forma cooperativa por equipos, con efecto se promueve en la innovación, se vuelve un tema fenomenológico.

Es importante que en el proceso autoevaluativo se caracteriza la reflexión, afirmando que todas las experiencias y vivencias, posibilitan un giro de la mirada, mediante el cual ellos mismos se convierten en objeto para la investigación o para el aprendizaje y por eso estas experiencias de aprendizaje, son por ello, fenómenos.

Por último, en la estructura de la Relatoría como "alma y corazón" de la innovación, se establece una sección de conclusiones, que establece la conjunción de las primeras cuatro secciones esenciales en lo referente al método o el cómo desarrolla las estrategias propias de cada sección. Igualmente, plantea el equipo de trabajo en cada proyecto, cuáles fueron las causas y los ¿por qué? Los Ilevaron a realizar dicha actividad y de la misma forma, se espera determinar las consecuencias o los para qué. Acá en estos requerimientos, se busca, hacer los registros: de lo simple a lo complejo, de los elementos al todo, de la causa a los efectos, del principio a las consecuencias.

\section{La evaluación un proceso de simulación que se atenúa en la innovación}

En este proceso se ha hecho mucho énfasis en tomar en las prácticas, como indicador y como criterio en la evaluación, los objetivos conductuales centrados en el estudiante como un intento de tipo operativo, de tal forma que se aplique el concepto evaluativo en congruencia con objetivos y producto entre procesos y aprendizaje, es decir, un continuo mirar de los avances y fracasos de los evaluados, de tal forma que se puedan hacer congruentes los resultados obtenidos con los objetivos propuestos $^{2}$, se puede decir en general que la evaluación es un concepto y componente de la escuela que tiene una condición polisémica, ya que se puede tomar como resultado, o también como procesos o como manejo de poder, permitiendo inferir una polivalencia en el desarrollo del trabajo evaluativo

2 La evaluación genera aprendizajes ¿desde la tradición o desde la innovación?. Oscar Colonia Alcalde. Enero 2012. 
Es claro que en la implementación de una propuesta de innovación y sobre todo en la disciplina de las matemáticas, se pueden dar unas relaciones de poder fuertes, ya que éstas por sí mismas manejan el poder que se tiene en la aplicación de los diferentes campos y contextos de la vida cotidiana como: la ingeniería, la economía, la medicina y por supuesto en la propia formación de maestros, lo que implica en la propia propuesta de innovación, ubicar las matemáticas desde una perspectiva de la etnomatemática o educación matemática crítica, creando un escenario en el aula, donde se desconcentre el poder del maestro (trabajo cooperativo en los dibujos), que permitan además, generar una más y mejor ubicación espacial de los estudiantes. Este escenario en el aula en un sistema de referencia (plano cartesiano) de saber y hacer (relatoría), de autoevaluación crítica de su aprendizaje y comprensión (diario pedagógico) y de transformación de su perspectiva del mundo (maqueta) Ileva a unas matemáticas con poder desde el saber y además poder hacer con el saber, que el estudiante deja de ser pasivo y acusmático en un aprendizaje pasivo y sin significado, a poder interactuar y relacionar situaciones matemáticas con lo social, cultural y propio de la ciencia. En el proceso evaluativo se busca conectar el mundo externo (vía el mundo de la vida) con el mundo propio del aula, buscando la generación de unas matemáticas más tangibles y menos abstractas. ${ }^{3}$

La evaluación en y desde la propuesta de innovación busca no convertirse como una "ley de instrumento", esto quiere decir que toda acción o práctica pedagógica no necesariamente debe ser evaluada porque entonces se caería en tomar la evaluación como un instrumento de experimentación e "instrumentación". La propuesta de innovación no considera al estudiante como un objeto de evaluación sino como un sujeto de evaluación, con su propia dinámica de desarrollo cognitivo y afectivo, una persona que debe exigir una importante significación y sentido en el aprendizaje del saber matemático.

De acuerdo a las premisas anteriores, la experiencia sobre la propuesta evaluativa se puede sintetizar en un decálogo que posibilita la optimización de los procesos cognitivos, procedimentales y axiológicos, buscando:

3 La evaluación genera aprendizajes ¿desde la tradición o desde la innovación?. Oscar Colonia Alcalde. Enero 2012. 
1. Evaluación del trabajo en equipo (acción de interacción en el aula).

2. Evaluación del trabajo en forma individual (acción- reflexión- sustentación de saberes).

3. Evaluación de la organización de la Relatoría (por unidades y secciones).

4. Evaluación de la relación teoría-práctica (ensayos-conceptos-procedimientos-proposiciones-reflexiones).

5. Evaluación de la actividad transformadora (procesos de conceptualización-análisis-relación-proposición).

6. Evaluación de la apropiación conceptual-procedimental (aplicación y transferencia).

7. Evaluación y formación autónoma del estudiante (autoevaluación en el saber, el hacer y el saber-hacer).

8. Evaluación de los procesos deductivos-inductivos y sintéticos (creatividad-hipótesis-conjeturas y conclusiones).

9. Potenciación de procesos (sugerencias y aportes sobre la forma y el fondo del proyecto matemático).

10. Acompañamiento a mejorar procesos por medio de recomendaciones y sugerencias.

\section{A manera de conclusión}

Mirar una innovación en el campo educativo, implica establecer transformaciones en: lo teórico, metodológico o evaluativo, o talvez en la conjunción de los tres, estableciéndose a nivel general lo siguiente:

- Si se establece que innovación es un cambio, se requiere mirar que mejorar ha llevado al contexto de aplicación y además, si ello implica algo, planeado, deliberado, reflexionado, sistematizado, etc., de lo contrario es sólo un cambio que se da en forma apariencial, ya que sería de tipo espontáneo.

- Tomaré la definición que Richland plantea sobre innovación (publicada por Moreno 1995) "La innovación es la 
selección, organización y utilización creativas de recursos humanos, materiales y superestructuras de maneras nuevas y propias que den como resultado la conquista de un nivel más alto con respecto a las metas y objetivos previamente marcados".

- Toda innovación y más en la disciplina matemática, implica una reflexión y sistematización de procesos en el aula con el propósito de transformar las estrategias y actividades que son obsoletas por la evolución del mundo, entonces, es necesario ubicar y organizar nuevas estrategias que impacten el hacer de los estudiantes, permitiendo el desarrollo de habilidades y el afinamiento de destrezas en el accionar del aula, promoviendo la visibilización de alumnos que no tienen capacidades propias para el saber matemático.

- Es necesario transversalizar, el saber matemático con el lenguaje, como condición para el mejoramiento comprensivo y conceptual, entonces, la teoría y los referentes que produjeron estas teorías, se ubican en la estructura de la innovación, buscando que el aprendizaje no sea mecánico, sino comprensivo de tal forma que la detonación de conceptos y procedimientos tengan una relación clara con los propósitos de una situación. Por ello las herramientas (dibujo-relatoríaetc.) se deben intersectar en forma permanente y continua con las actividades de cada sección o episodio, que determinen procesos transformadores que ayuden al análisis de nuevas concepciones.

- En los procesos innovadores se busca la autonomía de los alumnos, cuando en sus procesos a-didácticos configuran algunas ideas de peso que relacionan diferentes caminos que posibilitan la solución de un problema, con ubicaciones diversas en los marcos conceptuales que sustentan los procedimientos.

- La acción en el aula se diversifica y democratiza, cuando desde las diferentes actividades y estrategias se consolidan las aportaciones de los alumnos, potenciando así, el desarrollo de la unidad formativa, además, se consolida la autonomía de los actores, ya que muchas actividades se pueden realizar por medio de tareas que ellos mismos se colocan, ya que se requieren las consultas y pesquisas en internet y libros, generando un semillero de investigadores.

- En este proceso innovador se puede establecer que posibilita el aprendizaje del saber matemático ya que moviliza a 
los alumnos por diferentes marcos conceptuales, coloca en conflicto las creencias de los maestros y plantea inicialmente una resistencia mecánica al cambio, lo que ha permitido una parcial creencia de otros actores como alumnos y padres de familia al cambio paradigmático.

- El proceso innovador en el transcurso de diecisiete años ha seguido la dirección de "abajo-arriba", se presenta partiendo de una hipótesis principal y varias de tipo alternativo, las contradicciones y dificultades se toman como un proceso de configuración histórica y primordialmente la innovación abierta al contraste con otros colectivos de maestros desde un "debate orgánico".

\section{Referencias}

Aduriz, Agustín y otros (2002). Actualización en didáctica de las ciencias naturales y las matemáticas. Bogotá. Editorial Magisterio.

Bruer, Jhon T. Escuelas para pensar una ciencia del aprendizje en el aula. Buenos Aires: Paidós. Ministerio de Educación y Ciencia.

Carretero, Mario. Constructivismo y Educación. Buenos Aires. Aique. Primera Edición.

D'Amore, Bruno, Fandiño Martha I (2002). Un acercamiento analítico al "triángulo de la didáctica". Recuperado de http://www. dm.unibo.it/rsddm/it/articoli/damore/443\%20triangulo\%20de\% 20la\%20didactica.pdf

Camilloni, Alicia R. W. et al. La evaluación de los aprendizajes en el debate didáctico contemporáneo. Editorial Paidos.

Cantoral Ricardo et al. Desarrollo del pensamiento matemático. Editorial Trillas. Primera edición.

Colonia, O. (6 de Septiembre de 2008 a). Propuestas y posibles respuestas que permiten seguir con la apuesta. [Post en un blog]. Innovaciones y Ensayos en la Escuela. Recuperado de http://oscarcol.blogspot.com 
Colonia, O. (6 de Septiembre de 2008 b). Una mirada de reflexión sobre la innovación [Post en un blog]. Innovaciones y Ensayos en la Escuela. Recuperado de http://oscarcol.blogspot.com

Colonia, O. (6 de Septiembre de 2008 c). Una mirada histórica a nuestra vida, reflexa la historia y origen de nuestras acciones. [Post en un blog]. Innovaciones y Ensayos en la Escuela. Recuperado de http://oscarcol.blogspot.com

Colonia, O.(16 de Septiembre de 2007 a). Las matemáticas de la formalización a la contextualización. [Post en un Blog]. Innovaciones y Ensayos en la Escuela. Recuperado de http://oscarcol. blogspot.com

Colonia, O. (17 de Agosto de 2007 b). La circulación del conocimiento en la Escuela. [Post en un blog]. Innovaciones y Ensayos en la Escuela. Recuperado de http://oscarcol.blogspot.com

Gallego Badillo, Rómulo (1997). Discurso sobre el constructivismo. Santafé de Bogotá. Mesa Redonda Magisterio. Segunda edición.

Hernández, L. (5 de Octubre de 2012). "El Mundo de la Matemática en el Contexto de la Naturaleza" Por: Oscar Colonia. [Mensaje en un blog]. Diálogo entre profesores. Recuperado de https://http://dialogoentreprofesores.blogspot.com/2012/10/elmundo-de-la-matematica-en-el.html

Jaramillo. Juan Manuel, Duque, Luz Marina y otros. Thomas Kuhn. Santiago de Cali. Editorial Univalle.

Parra, Cecilia y Gaiz Irma. Didáctica de la matemática.

Vásquez, Rodriguez Fernando (2004). Pregúntale al ensayista. Editorial Kempis. Primera Edición. 